\title{
Determinación densidad de plantas/ha, en la siembra de ají paprika variedad Papriking para obtener campos con $80 \%$ de recojo y con daño mecánico menor al $25 \%$ en un proceso de cosecha mecanizada con cabezales de rotación invertida en Agrícola Cerro Prieto S.A.C
}

\section{Density determination of plants/ha, in the planting of air paprika variety Papriking for fields with $80 \%$ of pickup, and mechanical damage with less than $25 \%$ in a process of mechanized harvest heads with inverted rotation in Agricultural Cerro Prieto S.A. C}

Carlos Gozzer Puescas

\begin{abstract}
Escuela de Post Grado, Universidad Nacional de Trujillo, Av. Juan Pablo II S/N. Trujillo, Perú.
\end{abstract}
DOI: https://doi.org/10.33017/RevECIPeru2013.0015/

\section{Resumen}

El presente artículo titulado "Determinación densidad de plantas/ha, en la siembra de ají paprika variedad papriking para obtener campos con $80 \%$ de recojo y con daño mecánico menor al $25 \%$ en un proceso de cosecha mecanizada con cabezales de rotación invertida en Agrícola Cerro Prieto S.A.C." ha sido desarrollado con la finalidad de determinar la densidad de la siembra de ají paprika para lograr la mecanización de cultivo paprika. El trabajo de investigación se realizó en la Empresa Agrícola Cerro Prieto S.A.C, dedicada a la producción, empaque y comercialización de productos agrícolas ubicada en el norte del Perú, entre los valles de Jequetepeque y Zaña, y es producto del desarrollo de una irrigación 100\% privada que abarca 5,700 hectáreas [1]. Para el desarrollo del presente trabajo se ha utilizado la observación como técnica de recopilación de datos, lo cual sirvió como fuente de información para determinar el alcance del proyecto. Analizamos diferentes densidades de siembra para logrando determinar plantas/ha que permitan obtener campos con $80 \%$ de recojo. Para ello el presente trabajo fue de tipo experimental y se trabajó con 3 densidades distintas $(66,000 / 133,000 / 266,000)$ plantas /ha con irrigación de manguera simple y doble.

Como resultado de esta investigación podemos concluir que mediante la determinación de densidad $(266,000$ Plantas /ha) se puede obtener una mecanización de cosecha de ají paprika con un daño mecánico menor del $25 \%$.

Descriptores: papriking.

\section{Abstract}

This article entitled "Determining density of plants/ha in planting ají paprika variety papriking to obtain fields with $80 \%$ of pickup, and mechanical damage with less than $25 \%$ in a process of mechanized harvest heads with inverted rotation in agricultural cerro prieto S.A. C. " has been developed with the purpose to determine the density of planting of ají paprika to achieve the mechanization of crop paprika. The work of research was conducted at the Cerro Prieto Agricultural Company S.A.C, dedicated to the production, packaging and marketing of agricultural products located in the north of Peru, between the valleys of Jequetepeque and Zana, and is a product of the development of a $100 \%$ private irrigation that covers 5.700 hectares [1]. For the development of this work has been used as observation data collection technique, which served as a source of information to determine the scope of the project. We analyze different planting densities for achieving determine plants/ha to obtain fields with $80 \%$ of pickup. For this reason, the present work was experimental 
and was working with 3 different densities (66.000/133.000/266.000) plants/ha with irrigation of single and double hose. As a result of this investigation, we can conclude that using the density determination (266.000 plants/has) you can obtain a mechanization of crop of aji paprika with a mechanical damage less than $25 \%$.

Keywords: papriking.

\section{Introducción}

La empresa agroindustrial tiene una ventaja dentro de su producción, que es la gran demanda de productos como el pimiento páprika por el mercado extranjero [2], por ello están obligadas a sembrar más áreas de este cultivo, pero el alto costo y la falta de disponibilidad de mano de obra para la recolección se ha traducido en la disminución de la producción nacional, siendo menos competitivas para tomar posición de precio en el mercado internacional. La mayor parte de la cosecha sigue siendo cosechada a mano, el desplazamiento de la producción a lugares donde los costos laborales son bajos. La mecanización de la cosecha de Capsicum requerirá un enfoque de sistemas, integración de la investigación en mejoramiento de plantas, las densidades de producción y diseño de cosechadoras.

Agrícola Cerro Prieto enfocará todos sus esfuerzos en hacer viable la cosecha mecanizada a través de un estudio de investigación que ayude a Determinar la densidad de siembra (plantas/ha.), el cual permita obtener campos con $80 \%$ de recojo y con daño mecánico menor al $25 \%$ en un proceso de cosecha mecanizada con cabezales de rotación invertida.

\section{Método}

Como estrategia se trabajará con diferenciación por medio de su área de operaciones agrícolas método deductivo - analítico, desarrollando investigación acerca de estructurar una arquitectura de planta ideal que permita una cosecha mecanizada con el fin de bajar los costos de producción y enfrentar la falta de disponibilidad de mano de obra, lo cual nos ubicaría de manera competitiva y tomaríamos posición de precio en el mercado internacional.

\section{Procedimientos}

\section{Densidades de Cultivo}

Tabla 1. Densidades de Cultivo diferentes con uno y dos cintas de riego en los cultivo Páprika.

\begin{tabular}{|l|r|}
\hline Cintas & Densidad \\
\hline Con una manguera & 66,000 \\
& 133,000
\end{tabular}

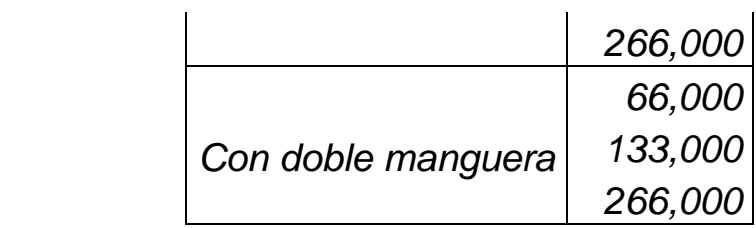

Manejo Agrícola

Tabla 2. Manejo agronómico para manguera siple y doble.

\begin{tabular}{|c|c|c|}
\hline Item & $\begin{array}{l}\text { Manguera } \\
\text { Simple }\end{array}$ & $\begin{array}{l}\text { Manguera } \\
\text { Doble }\end{array}$ \\
\hline Area Sembrada & 3.8 & 3.8 \\
\hline $\begin{array}{c}\text { Incorporación de } \\
\text { Guano }\end{array}$ & $30 \mathrm{t} / \mathrm{ha}$ & $30 \mathrm{t} / \mathrm{ha}$ \\
\hline Aplicación Ronstar & Sí & Sí \\
\hline Densidades & $\begin{array}{c}66,000 \\
/ 133,000 \\
/ 266,000 \\
\end{array}$ & $\begin{array}{c}66,000 \\
/ 133,000 \\
/ 266,000 \\
\end{array}$ \\
\hline Desahije & 30 DDS & 30 DDS \\
\hline $\begin{array}{c}\text { Aplicación } \\
\text { Hormonas }\end{array}$ & Paclobutrazol & $\begin{array}{c}\text { Etileno y/o } \\
\text { Paclobutrazol }\end{array}$ \\
\hline Disminución Riego & 180 días & 170 días \\
\hline Corte Riego & 185 días & 175 días \\
\hline $\begin{array}{c}\text { Aplicación Etileno } \\
\text { Defoliante }\end{array}$ & No & 180 días \\
\hline Cosecha a Máquina & No & 190 días \\
\hline
\end{tabular}

\section{Preparación de Terreno:}

- Arado: El objetivo de esta labor es voltear el suelo con la finalidad de exponer las capas sub superficiales a la superficie y de esta manera disminuir problemas de plagas; así como oxigenar el suelo. Se realizarán dos pasadas como mínimo (cruzadas) a una profundidad mínima de $35 \mathrm{~cm}$.

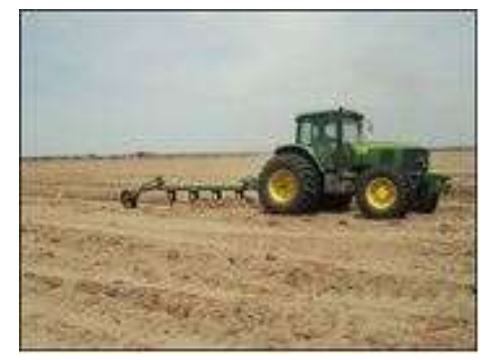

Imagen 1: Preparación de Terreno - Arado 
- Gradeo: El objetivo de esta labor es mullir el suelo hasta dejarlo suelto con la finalidad de poder realizar las labores posteriores (Surcado, encamado) sin dificultad y a la vez permitir un buen desarrollo del bulbo de humedad. La profundidad del gradeo debe ser mínimo $18 \mathrm{~cm}$.

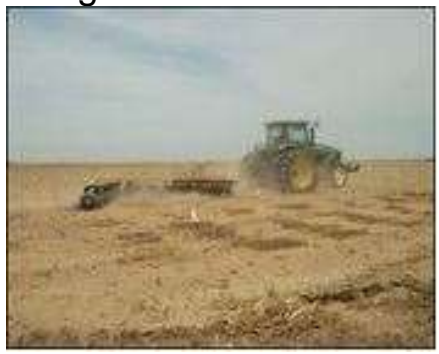

Imagen 2: Preparación de Terreno - Gradeo

- Pendoneo: El objetivo de esta labor es alinear los surcos de la línea de siembra o transplante y hacer que todos los surcos mantengan la misma distancia distancia.

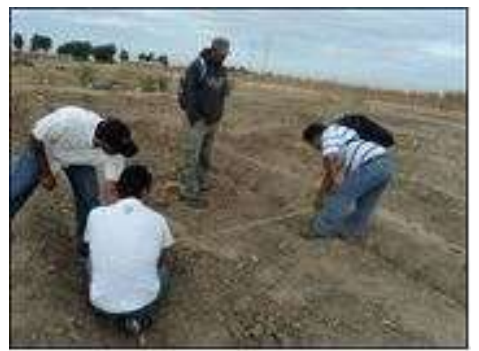

Imagen 3: Preparación de Terreno - Pendoneo

- Surcado: El objetivo de esta labor es abrir en el campo unas líneas a una profundidad de $18 \mathrm{~cm}$ y con una base de $10 \mathrm{~cm}$ en promedio que es donde finalmente se colocará la materia orgánica. Esta labor debe ser realizarse siempre usando el alineamiento de los pendones.

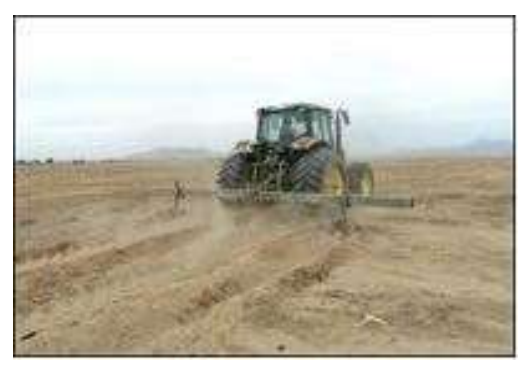

Imagen 4: Preparación de Terreno - Surcado

- Tapado y Mezcla de Guano: El objetivo de esta labor es tapar y mezclar el guano con la arena para que así la raíz del plantin no entre en contacto directo con este.

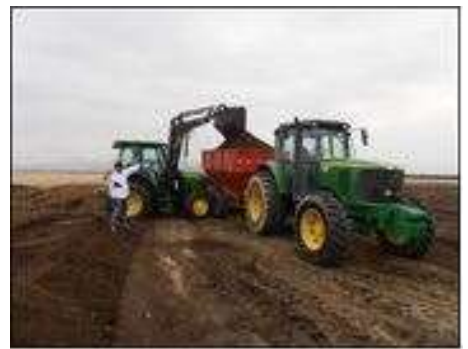

Imagen 5: Preparación de Terreno - Guano

- Encamado y Aplicación de Yeso: El objetivo de esta labor es realizar unas camas uniformes las cuales servirán de base o soporte el resto de vida del cultivo.

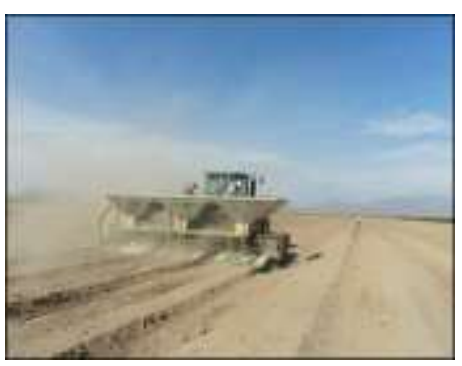

Imagen 6: Preparación de Terreno - Encamado

- Siembra Directa: Para cumplir los objetivos, se requiere provisión suficiente de agua para cumplir las exigencias de la germinación. Los vientos fuertes, cálidos y con carguío de arena ocasionan muerte por deshidratación de las semillas en germinación y de plántulas recién emergidas por lo cual tenemos que instalar cortinas de viento previo antes de la siembra.

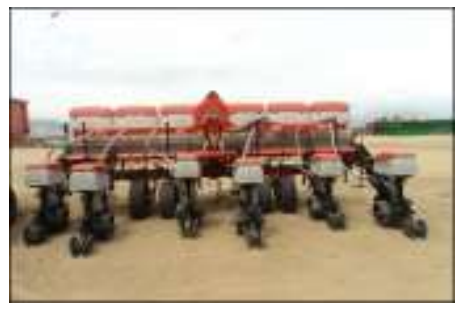

Imagen 7: Preparación de Terreno - Siembra

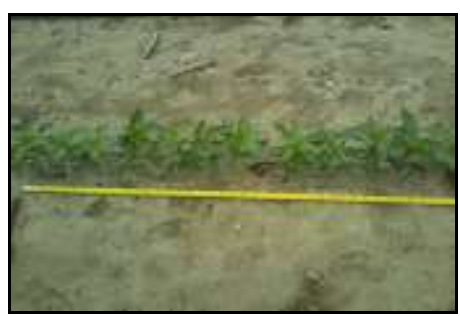

Imagen 8: Plantas de Paprika 
- Cultivador (Control de Maleza): El objetivo de esta labor es oxigenar e suelo para un buen desarrollo radicular así como eliminar las malezas que hayan emergido durante esta etapa.

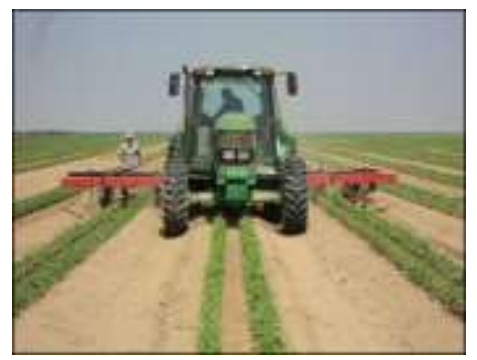

Imagen 9: Preparación de Terreno - Deshierbo

Época de Siembra: Los ajíes de exportación se pueden sembrar en la mayor parte del año, pero en la estación de verano es donde se presentan los mayores problemas $\mathrm{e}$ inconvenientes: mayor incidencia de plagas, enfermedades radiculares, abundancia de malezas, baja de rendimientos por altas temperaturas y a veces pérdidas por exceso de lluvias. En el caso del ají Morrón se ha observado una ligera sensibilidad a las bajas temperaturas de los meses más fríos (julio-agosto).

Factor a Cultivar: Hay algunas variaciones en cuanto al factor a cultivar empleado para cada tipo de ají, llámese el jalapeño, morrón, piquillo o paprika. Para el caso de este último la preferencia está dada sobre todo por el 'Papri King', aunque algunas empresas también siembran el cultivar "Sonora" que se presta para procesarlo como enlatado al estado verde.

Riego Sistema: Los sistemas de riego observados son dos: 1) riego por gravedad predominante en las áreas nuevas, las cuales están ubicadas sobre todo en las provincias de Ferreñafe y Chiclayo; y 2) riego por goteo que prima en la provincia de Lambayeque (distritos de Jayanca, Motupe y Olmos). El consumo de agua por gravedad es en promedio de 14,000 m3, mientras que por goteo se reduce aproximadamente a la mitad $(7,300 \mathrm{~m} 3)$. La frecuencia de los riegos en el sistema de gravedad es cada semana; mientras que en goteo es en promedio dos veces por día con tiempos de 20 minutos cada uno.14 Pozos y sistema de bombeo para riego de un productor pequeño.

\section{Control Fitosanitario:}

Este es el factor crítico de la producción, a donde están dirigidos los mayores esfuerzos y atención. Priman los problemas de plagas, pero el factor Enfermedades tampoco se puede descuidar. Como sucede en otros cultivos la incidencia de problemas fitosanitarios en Capsicum se acentúa en los meses de verano, obligando al productor a hacer aplicaciones más frecuentes.

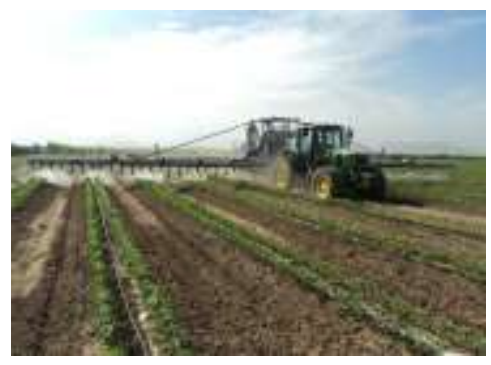

Imagen 10: Control Fitosanitario - Airtec

En cuanto a plagas insectiles las más comunes son: Symmetrischemacapsicum "polilla del aji", Prodiplosislongifila "mosquilla de los brotes", Lineodes integra "gusano pegador de las hojas", Spodopteraeridania "gusano del follaje", Bemisiatabasci "mosca blanca", Myzuspersicae "pulgones". Una plaga ocasional también es Ceratitiscapitata "mosca de la fruta" que ataca a los productos de cosecha. Respecto a enfermedades, las que más pueden incidir son virosis, marchitez por hytophthoracapsici, oidiosis por Leveillulataurica y particularmente en la zona de Zaña el hielo o rancha causado por Phytophthorainfestans. Los productores líderes aplican todas las estrategias del manejo integrado de plagas, aparte de pesticidas utilizan el control etológico: trampas amarillas, blancas, negras, trampas de luz, trampas de feromonas, etc [3].

\section{Cosecha: (Boese $420 \mathrm{C})$}

Objetivo: Poder permitir que las empresas puedan programar y realizar sus cosechas con el número de Hectáreas demandadas por el mercado sin tener la limitante de disponibilidad de mano de obra.

Reducir los costos de cosecha de páprika logrando ahorros de hasta 2500 \$/ha.

\section{¿Cómo lo logramos?}

Para lograr la cosecha mecanizada hemos tenido que hacer una investigación con ensayos primero realizando modificaciones a la cosechadora Boese 420c, con el fin de hacer viable la cosecha mecánica en Páprika y calibrando la máquina conociendo su comportamiento en cosecha logrando diseñar e implementar algunas modificaciones dentro de la cosechadora de páprika, la cual se ilustrara en 5 etapas: 
- Primera Etapa: Inicialmente se probó la cosechadora, realizando las siguientes calibraciones:

Altura de cabezal: $10 \mathrm{~cm}$ del suelo.

Distancia media entre helicoidales: $5 \mathrm{~cm}$

Velocidad de trabajo: $1.5 \mathrm{~km} /$ hora.

Distancia entre cabezales: $80 \mathrm{~cm}$.

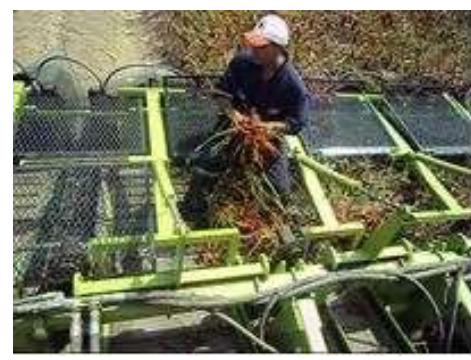

Imagen 11: Cosechadora Boese

- Segunda Etapa: Después de la primera etapa y frente a los atoramiento mostrados de PPK consideramos las siguientes modificaciones.

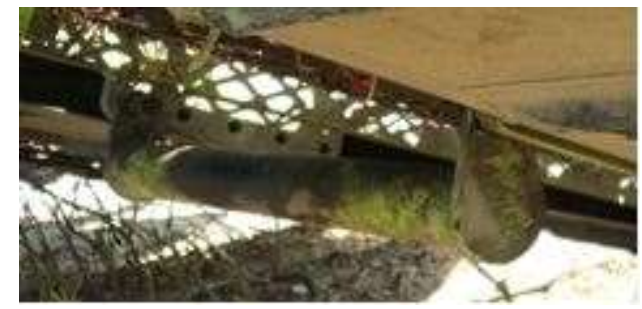

Imagen 12: Cambio en la posición de la barra echadora de planta.

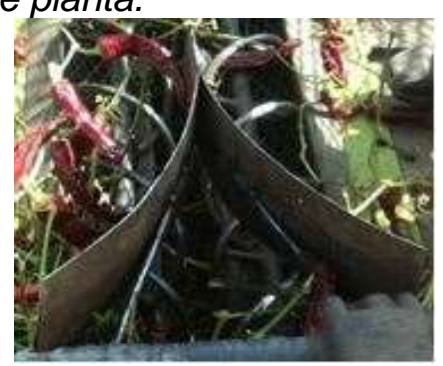

Imagen 13: Construcción de separador y cortador de plantas (chatin).

Se observó atoramiento en la parte superior de los helicoidales.

La altura de la planta supera a la altura de trabajo del cabezal de cosecha.

El trabajo del separador de planta es bueno pero quita espacio para el paso de la planta, lo cual produce atoramiento lateral ocasionado por las ramas y frutos.
- Tercera Etapa: Después de las modificaciones se observó problemas con los helicoidales y se procedió hacer los siguientes cambios

Se recortó $15 \mathrm{~cm}$ del tramo superior de 4 helicoidales (2 líneas de cosecha), con la finalidad de dejar un espacio libre por donde pase la planta y evitar atoros en los extremos superiores de los cabezales de cosecha.

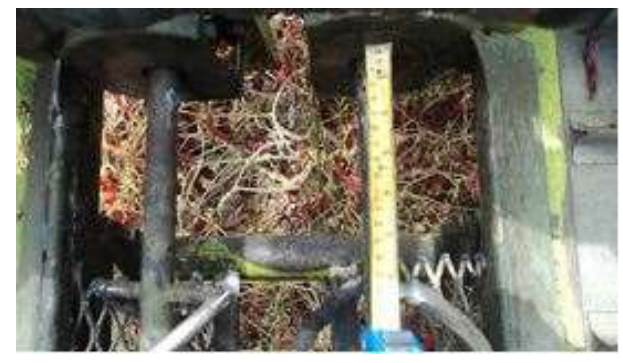

Imagen 14: Atoro en Cabezales de Cosechadora

El resto de helicoidales no fueron modificados, para comparar resultados.

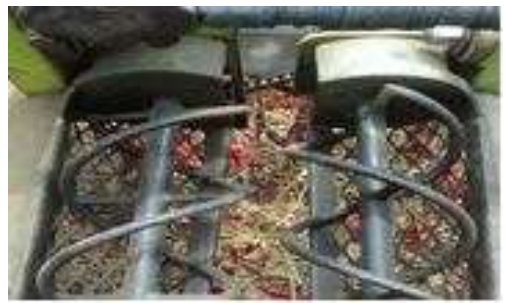

Imagen 15: Helicoidales de Cosechadora

El cabezal de cosecha se calibró verticalmente (máximo ángulo de cosecha)

Se alinearon los puntones de tal manera que quedaran justo en el centro de las líneas de siembra.

Se determinó que la velocidad adecuada de la cosechadora sería de $1 \mathrm{~km} / \mathrm{h}$ para realizar el ensayo.

Se calibró el espacio entre helicoidales, separándolos al máximo:

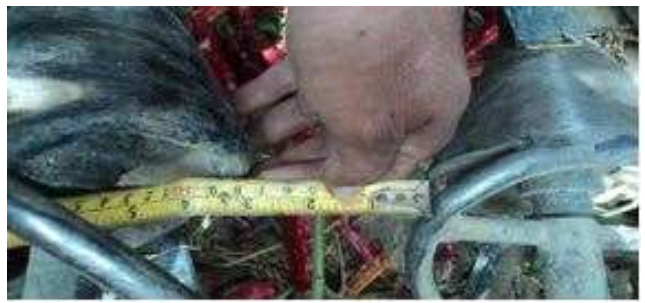

Imagen 16: Helicoidal - Extremo Inferior $7.5 \mathrm{~cm}$ 


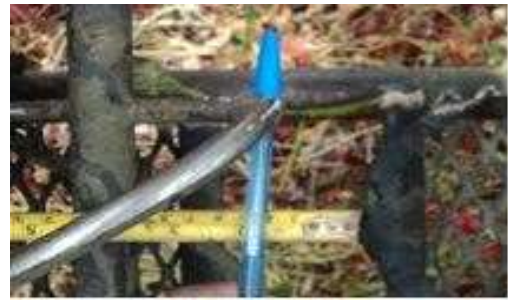

Imagen 17: Helicoidal - Extremo Superior $4 \mathrm{~cm}$

Se observó, que en los helicoidales que fueron recortados se redujo la cantidad de ramas atoradas, con respecto a los normales.

La apertura al máximo entre helicoidales facilitó el paso de las ramas, disminuyendo el atascamiento, pero dejando los frutos centrales de la planta.

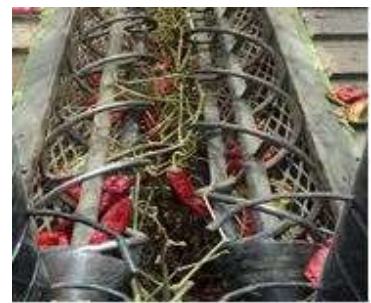

Imagen 18: Helicoidal con apertura máxima

Con altura de plantas superior a la del cabezal ocasiona que se enriende las plantas en los helicoidales.

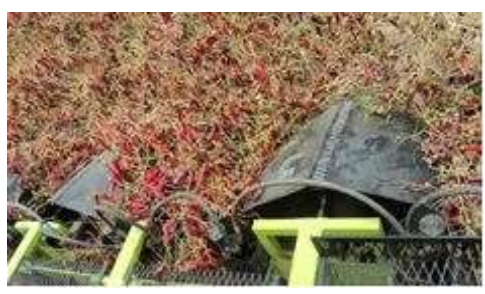

Imagen 19: Plantas con altura superior al Cabezal

Coordinaremos con producción para obtener la dosis y el volumen adecuado para la defoliación como el determinar el día de corte de agua a fin de evitar que los frutos sean botados rápidamente por los cabezales del a máquina.

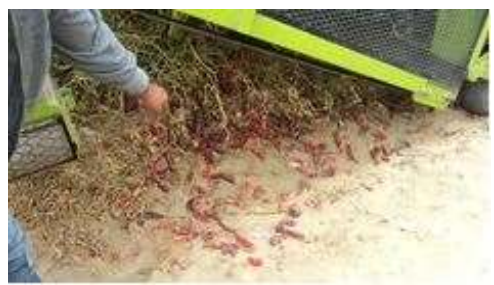

Imagen 20: Frutos Botados por los Cabezales
- Cuarta Etapa: Considerando que continuaba el atoramiento de las ramas de PPK en la tercera etapa optamos por construcción de Hélices cortadora de ramas.

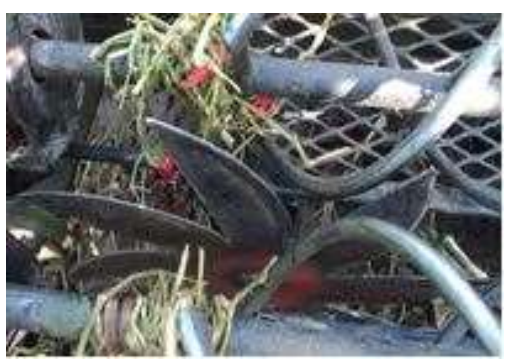

Imagen 21: Fabricación de hélice Vertical

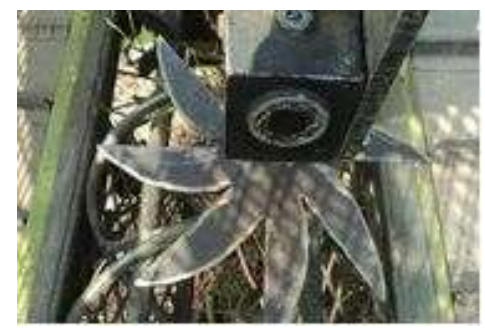

Imagen 22: Fabricación de hélice Horizontal

La hélice horizontal corta las ramas en buena proporción, amontona las ramas al sentido de giro y causa mucho daño mecánico a los frutos.

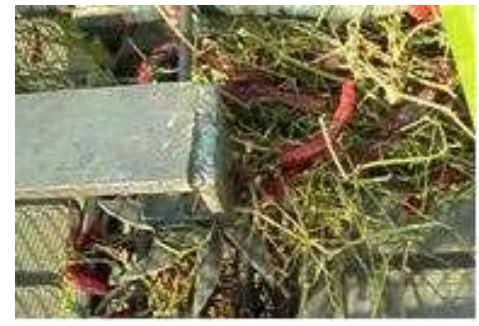

\section{Imagen 23: Plantas Amontonadas en Hélice} Horizontal

El disco de corte vertical presenta mejor eficiencia que el disco horizontal en lo que respecta a evitar el atoramiento pero aumenta el porcentaje de frutos rotos a un $30 \%$.

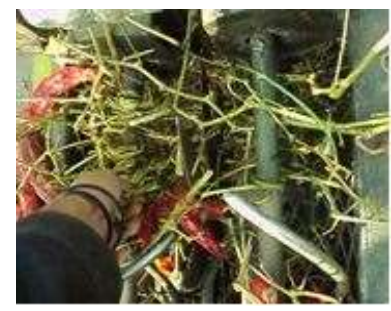

Imagen 24: Plantas cortadas por Hélice Vertical 
- Quinta Etapa: Frente a que continuamos con los atoramientos por ramas hasta la $4^{\circ}$ etapa es que tomamos la decisión de cambiar la posición de los motores hidráulicos.

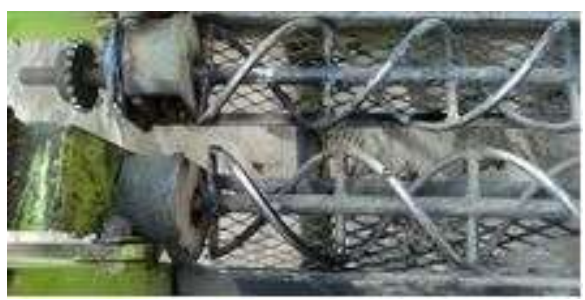

Imagen 25: Soldado de parte Recortada de helicoidales en la prueba $N^{\circ} 03$.

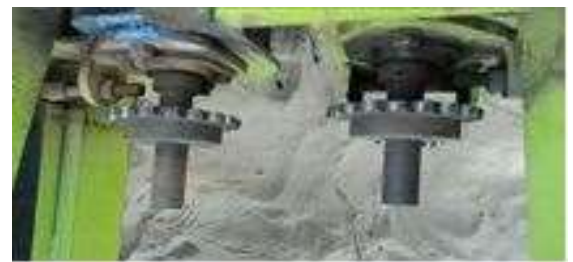

Imagen 26: Alargargamiento de cadena de transmisión de cabezales

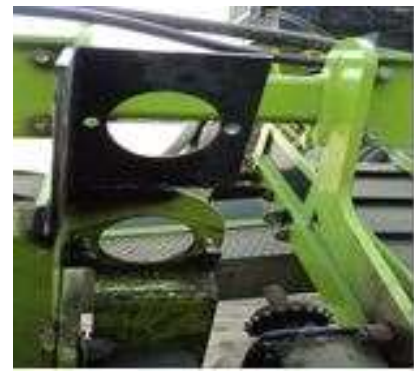

Imagen 27: Levantar motor hidráulico de cabezal de cosecha.

Etapa Final:

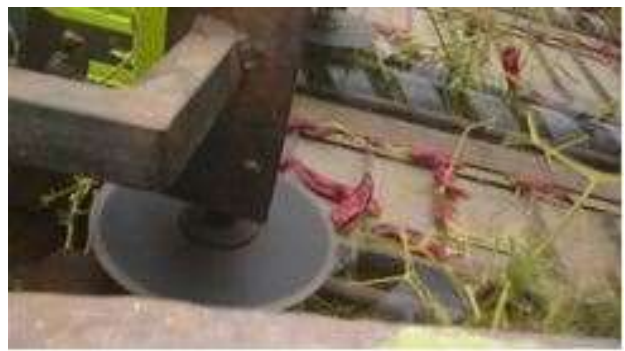

Imagen 28: Corte de Plantas en Cabezales

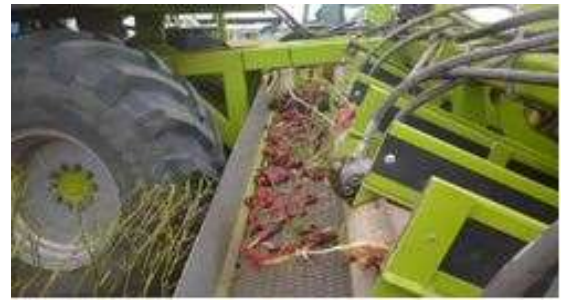

Imagen 29: Faja Transportadora de Frutos

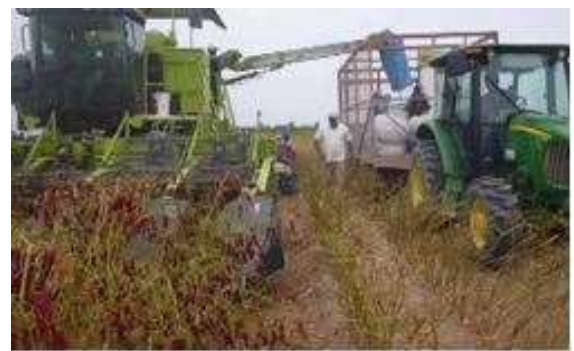

Imagen 30: Recepción Final de Producto Cosechado

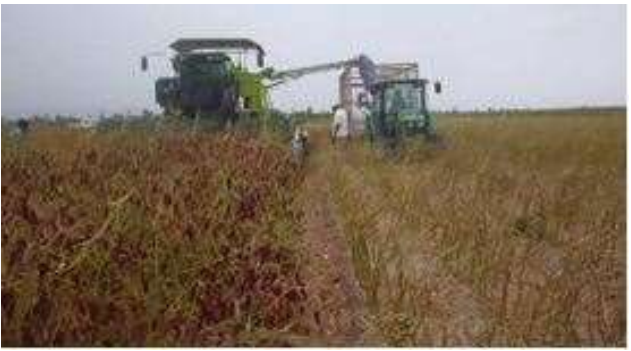

Imagen 31: Vista Final de Cosecha Mecanizada de paprika

\section{Resultados:}

Tabla 3: Resultados de Cosecha Mecanizada

\begin{tabular}{|c|c|c|c|}
\hline $\begin{array}{c}\mathrm{Kg} \\
\begin{array}{c}\text { Cosechados } \\
\text { por Maquina }\end{array}\end{array}$ & $\begin{array}{c}\mathrm{Kg} \\
\text { Cosechados } \\
\text { Manual }\end{array}$ & $\begin{array}{c}\% \\
\text { Cosechado }\end{array}$ & $\begin{array}{c}\% \\
\text { Entero }\end{array}$ \\
\hline 3,133 & 1,122 & $74 \%$ & $79 \%$ \\
\hline 1,800 & 605 & $75 \%$ & $76 \%$ \\
\hline 2,540 & 777 & $77 \%$ & $80 \%$ \\
\hline 2,471 & 895 & $73 \%$ & $77 \%$ \\
\hline
\end{tabular}

Capacidad de Cosecha mecanizada actual 1.2 Ha/Día. Y la meta es llegar a 2 Ha/Día para esto necesitaríamos contar acondicionar un centro de mantenimiento móvil en la cosecha con un Kit de repuestos para evitar tiempos muertos por paradas de maquina en la cosecha.

Hemos logrado alcanzar un $78 \%$ promedio de frutos enteros y la meta es llegar alcanzar un $90 \%$ de frutos enteros ensayando con diferentes tipos de geometría de discos de corte y la encontrar la 
posición optima con respecto a la helicoidal de la maquina.

\section{Tabla 4: Producción Según Uso de Mangueras}

\begin{tabular}{|c|c|}
\hline Manguera Simple & 7,186 \\
\hline Manguera Doble & 7,455 \\
\hline
\end{tabular}

Tabla 5: Porcentaje de recojo Según Uso de Mangueras

\begin{tabular}{|c|c|}
\hline Manguera Simple 66,000 & $69 \%$ \\
\hline Manguera Simple 133,000 & $76 \%$ \\
\hline Manguera Simple 266,000 & $76 \%$ \\
\hline Manguera Doble 66,000 & $78 \%$ \\
\hline Manguera Doble 133,000 & $81 \%$ \\
\hline Manguera Doble 266,000 & $79 \%$ \\
\hline
\end{tabular}

Tabla 6: Porcentaje de Frutos Rotos por Cosecha

\begin{tabular}{|c|c|c|c|c|}
\hline \multicolumn{5}{|c|}{ Porcentaje de frutos rotos debido a cosecha } \\
Variedad & Dendidad & $\begin{array}{c}\text { Fruto } \\
\text { Roto } \\
\%\end{array}$ & $\begin{array}{c}\text { Fruto } \\
\text { Entero } \\
\%\end{array}$ & $\begin{array}{c}\text { Total } \\
\%\end{array}$ \\
\hline \multirow{3}{*}{$\begin{array}{c}\text { Manguera } \\
\text { Simple }\end{array}$} & 66,000 & 23 & 77 & 100 \\
\cline { 2 - 5 } & 133,000 & 23 & 77 & 100 \\
\cline { 2 - 5 } & 266,000 & 22 & 78 & 100 \\
\hline \multirow{2}{*}{$\begin{array}{c}\text { Manguera } \\
\text { Doble }\end{array}$} & 66,000 & 20 & 80 & 100 \\
\cline { 2 - 5 } & 133,000 & 17 & 83 & 100 \\
\cline { 2 - 5 } & 266,000 & 19 & 81 & 100 \\
\hline
\end{tabular}

Tabla 4: Jornales Utilizados por Hectárea

\begin{tabular}{|c|c|c|c|c|}
\hline \multicolumn{5}{|c|}{ Numero de Jornales Aproximados por Ha } \\
Empleados por Fecha y Actividad \\
Fecha & Recojo & Selección & $\begin{array}{c}\text { Recojo } \\
\text { en } \\
\text { Cartera }\end{array}$ & Total \\
\hline $15-n o v$ & 21 & 6 & 2 & 29 \\
\hline $17-n o v$ & 21 & 4 & 1 & 26 \\
\hline $18-n o v$ & 24 & 4 & 1 & 26 \\
\hline $19-n o v$ & 22 & 5 & 1.5 & 28.5 \\
\hline
\end{tabular}

Tabla 6: Rendimientos de Cultivo

\begin{tabular}{|c|c|c|}
\hline Costos & $\$$ & $\$ /$ ha \\
\hline Costo Cultivo incluido Cosecha & 8,000 & 1,07 \\
\hline Costo Secado & 1,125 & 0,15 \\
\hline Costo Empaque Prensa & 525 & 0,07 \\
\hline Costo Tota & 9,650 & 1.29 \\
\hline
\end{tabular}

Teniendo en cuenta que nuestro rendimiento en $\mathrm{Kg}$ fue de $7,500 \mathrm{Kg} / \mathrm{Ha}$.

\section{Conclusiones}

De la presente investigación se desprenden una serie de conclusiones relevantes no tan sólo para entender el papel de las empresas agroindustriales, sino, incluso, para valorar el nivel de mecanización actual y aporte en el sector agrario.

Según los antecedentes las empresas agroindustriales en el Perú dedicadas a la siembra de ají páprika han aumentado las hectáreas de producción a la gran demanda originada por el mercado extranjero por lo que podemos concluir que la determinación de la densidad para una buena cosecha mecanizada, servirá para la empresa Agrícola Cerro Prieto S.A.C. tenga un crecimiento sostenido, para encarar a la competencia, consolidar su permanencia en el mercado y obtener ventajas competitivas frente a otras empresas de siembra de capsicum.

Sin duda alguna la situación actual de la demanda mano de obra con los nuevos proyectos de irrigación de las empresas agroindustriales va en aumento, por lo que el crecimiento en el sector agroindustrial reducirá la captación de personal para las campañas de cultivos por ello en definitiva se requiere permita una cosecha mecanizada a fin de lograr enfrentar la falta de mano de obra y ubicar de manera competitiva a nuestra empresa y sobre todo posicionar precio en el mercado internacional.

De igual manera tenemos la conclusión de la hipótesis se puede lograr la mecanización de la cosecha con un $83 \%$ de frutos enteros sin daño y 17 $\%$ de frutos con daño mecánico.

\section{Agradecimientos}

A mis hijas Nicolle y Cielo que son ellas mi razón de ser en este mundo y que deseo que tomen mis éxitos como un camino a seguir, en su vida futura.

\section{Referencias}

[1] ACP Natural, Nuestra Empresa, Disponible en: http://www.agricolacerroprieto.com

[2] J. C. León Carrasco, Precio de exportación de páprika mejoraría en abril (citado 15 enero 2013), Disponible en:

http://agraria.pe/noticias/precio-de-exportacionde-paprika-mejoraria-en-abril

[3] Trading Consult, mejora de las técnicas y procesos en la producción, cosecha y acopio de ajíes en Lambayeque (citado Setiembre 2009), Disponible en:

http://www.mincetur.gob.pe/Comercio/ueperu/li citacion/pdfs/Informes/9.pdf

E-mail:cgozzer@gmail.com 